\title{
OSTEOPOROSE COMO PREDITOR DE FRATURAS DO COLO FEMORAL POR QUEDAS EM IDOSOS: REVISÃO INTEGRATIVA
}

\author{
OSTEOPOROSIS AS PREDICTOR OF FEMORAL COLLAGE FRACTURES
}

BY FALLS IN ELDERLY: INTEGRATIVE REVIEW

\author{
Katyene dos Santos Medrado (MEDRADO, K. S.) \\ Discente do curso de Fisioterapia, Faculdade Evangélica de Ceres. Ceres-GO, Brasil. \\ katy.medrado@gmail.com
}

\author{
Maísa Moura Vieira (VIEIRA, M. M.) \\ Discente do curso de Fisioterapia, Faculdade Evangélica de Ceres. Ceres-GO, Brasil. \\ maysamoura25@gmail.com
}

\author{
Suelen Marçal Nogueira (NOGUEIRA, S. M.) \\ Doutora em Ciências da Saúde-FM/UFG \\ Docente da Faculdade Evangélica de Ceres. Ceres-GO, Brasil. \\ suelen.nogueira@fecer.edu.br
}

Endereço para correspondência: Av. Brasil, S/N, Qd. 13, Setor Morada Verde, 21 Ceres GO, Brasil. CEP: 763000-000 Fone: (62) 3323-1040

\section{RESUMO}

Introdução: Em virtude do envelhecimento podem surgir patologias como a osteoporose que atua na redução da massa óssea, favorecendo idosos a ficarem mais suscetíveis a sofrerem quedas e possivelmente fraturas, principalmente do colo femoral. A intervenção cirúrgica precoce no pós-fratura é imprescindível, pois a demora no tempo de internação e realização das cirurgias podem aumentar as taxas de letalidade. Objetivo: Verificar se a osteoporose é um fator predisponente para fraturas do colo femoral por quedas em idosos, se o gênero pode influenciar na progressão da patologia, além das mortalidades. Metodologia: Trata-se de uma revisão integrativa da produção científica, com buscas realizadas na biblioteca virtual da saúde (BVS), com inclusão da LILACS, MEDLINE, e na Cochrane Library no período compreendido entre 2015 e 2020, sendo incluídos 8 estudos nos idiomas inglês, português e espanhol para análises. Resultados e discussão: Foram utilizados diferentes instrumentos como: densitometria óssea, Fracture Risk Assessment Tool (Frax), questionários, software Trabecular Bone Score (TBS), e Espectroscopia Dispersiva de raios-x (EDS) para avaliação dos indivíduos, a osteoporose foi observada na maioria dos pacientes, porém nem todos 
tinham conhecimento do diagnóstico, o gênero feminino prevaleceu devido a diminuição dos níveis de estrogênio. Nas fraturas, as quedas se destacaram, 66,43\% necessitaram de apoio instrumental, e 11\% foram à óbito dentro de um ano. Conclusão: A osteoporose pode ser considerada uma preditora de fraturas do colo femoral em idosos, principalmente em mulheres, observa-se a necessidade de mais estudos com populações específicas, que evidenciem tais episódios podendo levar a morte.

Palavras-chave: Osteoporose. Idoso. Fraturas do colo femoral. Cirurgia.

\begin{abstract}
Introduction: Due to aging, pathologies such as osteoporosis may appear which acts in the reduction of bone mass, favoring the elderly to become more susceptible to suffering falls and possibly fractures, especially of the femoral neck. Early surgical intervention in the postfracture is essential, as the delay in hospitalization and surgery can increase lethality rates. Objective: To verify whether osteoporosis is a predisposing factor for femoral neck fractures due to falls in the elderly, whether gender can influence the progression of the pathology, in addition to mortality. Methodology: This is an integrative review of scientific production, with searches carried out in the virtual health library (VHL), including LILACS, MEDLINE, and the Cochrane Library in the period from 2015 and 2020, including 8 studies in English, Portuguese and Spanish for analysis. Results and discussion: Different instruments were used, such as bone densitometry, Fracture Risk Assessment Tool (Frax), questionnaires, Trabecular Bone Score (TBS) software, and X-ray Dispersive Spectroscopy (EDS) to assess individuals, osteoporosis was observed in most patients, but not all were aware of the diagnosis, the female gender prevailed due to the decrease in estrogen levels. In fractures, falls stood out, $66.43 \%$ needed instrumental support, and $11 \%$ died within a year. Conclusion: Osteoporosis can be considered a predictor of femoral neck fractures in the elderly, especially in women, there is a need for further studies with specific populations, which show such episodes, which can lead to death.
\end{abstract}

Key words: Osteoporosis. Elderly. Fractures of the femoral neck. Surgery.

\title{
1. INTRODUÇÃO
}

O envelhecimento populacional vem sofrendo alterações morfológicas e funcionais, aumentando as demandas dos serviços e ônus para a saúde com ocorrências de doenças crônicas não transmissíveis e a maior parte está relacionada ao aumento da perda da capacidade funcional (BARRETO; CARREIRA; MARCON, 2015; SILVA et al., 2018).

Com o envelhecimento e reabsorção óssea, há uma diminuição da massa e, quanto mais intensos, maiores as chances de aparecimento de patologias como a osteoporose. Sendo definida como uma doença osteometabólica que ocasiona degradação estrutural do tecido ósseo e redução da densidade mineral com aumento da fragilidade esquelética (CESAR et al., 2020; CURTIS et al., 2017; SILVA; ANDRADE; AMARAL, 2015). 
Devido à deficiência de estrogênio, a pós-menopausa do tipo I pode ser dividida em primária e fisiológica, ocorrendo geralmente após os 50 anos e a do tipo II senil que acomete sujeitos a partir dos 70 anos decorrentes de diminuição da atividade osteoblástica; e a secundária ocorre quando outras doenças (endocrinológicas, distúrbios renais, deficiências nutricionais) afetam a massa óssea (TOMMASO et al., 2016).

É uma patologia que atinge 200 milhões de indivíduos em todo mundo. Estima-se que $30 \%$ a $50 \%$ de mulheres passam por tal problema, aproximadamente $40 \%$ brancas e $13 \%$ dos homens com idade superior a 50 anos sofrem ao menos alguma fratura osteoporótica no decorrer dos anos (SCHURMAN et al., 2017).

O diagnóstico em estágio inicial diminui as chances de episódios de fraturas e morbimortalidade. O exame de densitometria analisa o grau de perda de massa óssea, podendo ser classificados em osteopenia com escores $\mathrm{T}$ entre $-1,0$ a $-2,5$, osteoporose menor que $-2,5$ ou como normais maiores que -1,0 em desvio padrão (LIU et al., 2015; MUNHOZ et al., 2018; NETO et al., 2015).

Acredita-se que a diminuição da massa óssea provocada pela osteoporose, favorece a ocorrência de fraturas do colo femoral em consequência de quedas que são muito recorrentes em idosos, e que acontecem nos próprios domicílios, pois correspondem em torno de $35 \%$ das intervenções ocasionadas por traumas (SPINELLI et al., 2018; VALLES-FIGUEROA et al., 2015).

Um estudo realizado por Singer e colaboradores (2015) revelaram que 4,9 milhões de pessoas foram hospitalizadas por fraturas osteoporóticas entre 2000 e 2011, correspondendo a mais de $40 \%$ das internações. A taxa de mortalidade chega a $11,9 \%$, devido ao tempo de internação e atraso das cirurgias com probabilidades de infecções hospitalares (EDELMUTH et al., 2018; HAGINO et al., 2017).

Portanto, com a dimensão de indivíduos portadores de osteoporose e das crescentes taxas de mortalidade de idosos após quedas e fraturas do colo femoral, a presente revisão integrativa teve como objetivo investigar se essa patologia pode ser considerada um fator relevante para tais eventos, se o gênero é influenciável bem como verificar as mortalidades.

\section{METODOLOGIA}

O estudo trata-se de uma revisão integrativa da produção científica, com levantamentos de dados nos bancos da BVS (Biblioteca Virtual da Saúde), com inclusão da 
LILACS (Literatura Latino-Americana e do Caribe em Ciências da Saúde) e MEDLINE (National Library of Medicine) e na Cochrane Library.

As buscas foram realizadas com o cruzamento dos descritores "osteoporose", "idoso", "fraturas do colo femoral" e "cirurgia", todos confirmados como Descritores em Ciências da Saúde no DeCs da Biblioteca Virtual de Saúde e no MeSH (Medical Subject Headings) utilizando para combinação os operadores boleanos "And" e "Or".

\begin{tabular}{|c|}
\hline BVS \\
\hline $\begin{array}{l}\text { ((osteoporose) or (osteoporose senil) and (idoso) or (pessoas idosas) and (fraturas do colo } \\
\text { femoral) and (cirurgia) or (operações)). }\end{array}$ \\
\hline Cochrane Library \\
\hline $\begin{array}{l}\text { ((osteoporosis or senile osteoporosis) and (Aged or old people) and (femoral neck fractures) } \\
\text { and (surgery or operations)). }\end{array}$ \\
\hline
\end{tabular}

Os critérios de inclusão para seleção dos artigos foram: (a) pesquisas que relataram a osteoporose como um fator para quedas e fraturas do colo femoral; (b) artigos com população $\geq 60$ anos de idade; (c) artigos nos idiomas inglês, português ou espanhol; (d) estudos do tipo coorte; (e) artigos publicados entre os anos de 2015 e 2020.

A definição dos últimos 5 anos foi em virtude de averiguar o que a literatura traz de mais atual sobre o tema proposto. Foram excluídos: (a) artigos duplicados; (b) estudos não disponíveis na íntegra; (c) artigos com textos incompletos; (d) estudos em animais. A seleção dos estudos sucedeu por rastreamento de títulos, análise dos resultados, após, selecionados por meio dos textos completos para uma leitura exploratória seguida de uma analítica e interpretativa.

Para distribuição e organização dos estudos, foi utilizado o fluxograma do protocolo prisma que consiste basicamente em quatro etapas (identificação, seleção, elegibilidade e inclusão) (Fluxograma 1) (GALVÃO; PENSANI; HARRAD, 2015). Para análise estatística descritiva, os dados foram inseridos em planilhas usando o software Excel®, para obtenção dos resultados em relação ao gênero e achados da osteoporose como preditora de fraturas por quedas, apresentados em forma de quadro e gráfico. 
Fluxograma 1- Busca e seleção de publicações científicas da osteoporose como preditora de fraturas do colo femoral (protocolo prisma).

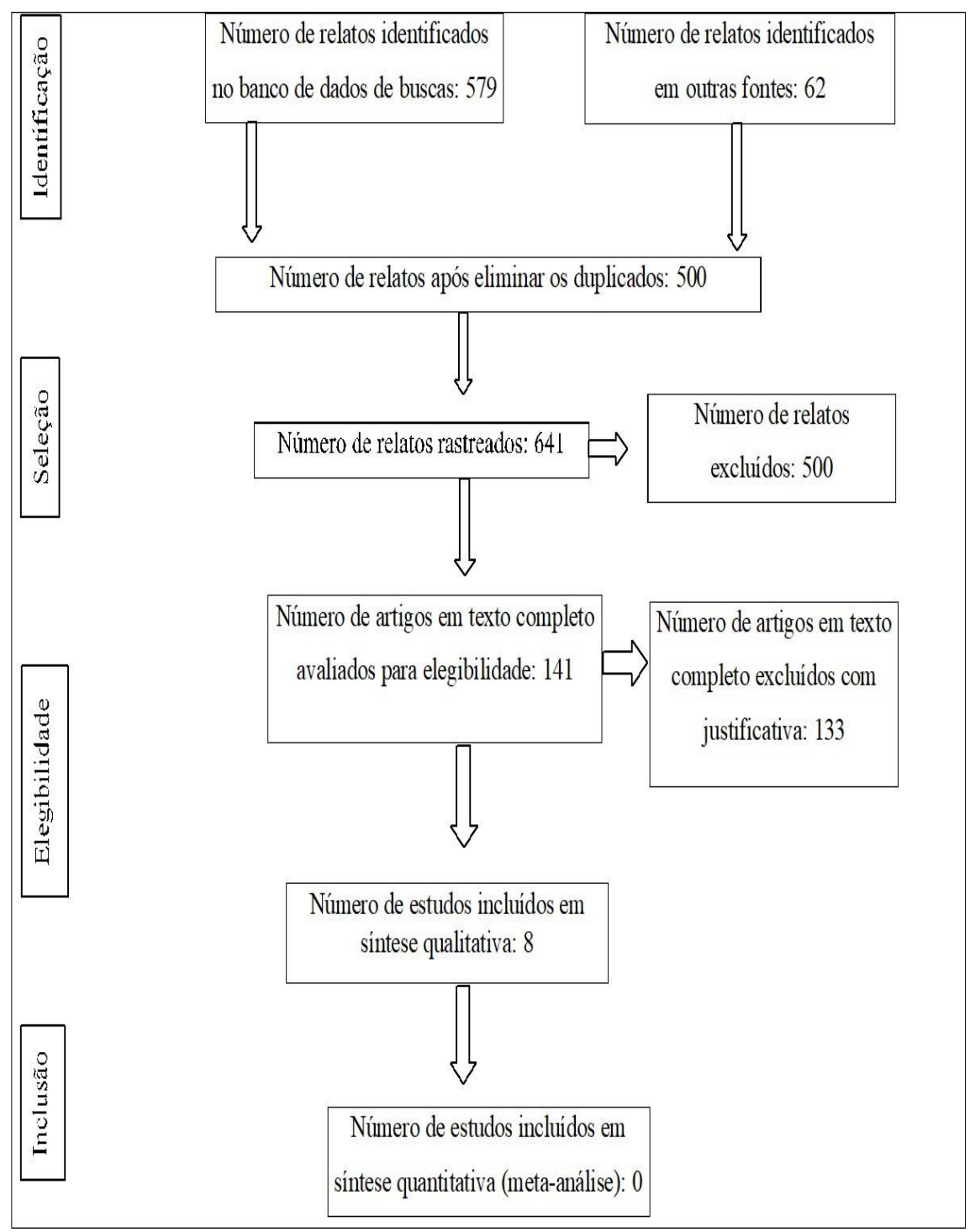

Fonte: GALVÃO, T.F.; PANSANI, T. S. A.; HARRAD, D. - Principais itens para relatar Revisões sistemáticas e Meta-análises: A recomendação PRISMA; Epidemiologia e Serviços de Saúde. 2015.

\section{RESULTADOS E DISCUSSÃO}

Dos 8 estudos selecionados todos foram publicados e indexados ao Portal de Periódicos da Capes (base eletrônica de dados de produções científicas), com Qualis entre A1 e B2. Sendo que $25 \%$ foram produzidos na América do Sul, Brasil e Uruguai, 37,5\% na América do Norte, Canadá e Estados Unidos, 25\% no continente Asiático, Turquia e Japão, e12,5\% na Europa. Utilizaram-se diferentes instrumentos de avaliação para identificação da osteoporose e constatá-la como preditora de fraturas por quedas na população idosa (quadro1). 
Quadro 1- Estudos que correlacionam os instrumentos empregados com a osteoporose como preditora de fraturas por quedas, publicados entre 2015 e 2020.

\begin{tabular}{|c|c|c|c|}
\hline Autor, ano & Instrumentos & $\begin{array}{c}\text { Achado } \\
\text { sobre } \\
\text { osteoporose } \\
\text { preditora de } \\
\text { fraturas por } \\
\text { quedas? }\end{array}$ & Conclusão \\
\hline $\begin{array}{l}\text { Adachi et } \\
\text { al., } 2019\end{array}$ & $\begin{array}{c}\text { Questionários com dados } \\
\text { sóciodemográfico, condições } \\
\text { médicas, histórico familiar de } \\
\text { fraturas, quedas, uso de } \\
\text { medicamentos, ingestão } \\
\text { alimentar, estilo e qualidade } \\
\text { de vida, altura e peso } \\
\text { associado com o exame de } \\
\text { densitometria óssea. }\end{array}$ & Sim & $\begin{array}{l}\text { O risco de fraturas iminente } \\
\text { entre mulheres idosas com } \\
\text { osteoporose, baixa massa } \\
\text { óssea ou histórico de fratura é } \\
\text { maior entre aquelas com } \\
\text { histórico de quedas, fratura } \\
\text { por baixo trauma e pior } \\
\text { função física. }\end{array}$ \\
\hline $\begin{array}{c}\text { Iki et al., } \\
2015\end{array}$ & $\begin{array}{l}\text { Software TBS, para avaliar a } \\
\text { microarquitetura óssea, } \\
\text { FRAX e entrevista para } \\
\text { informações como idade } \geq 65 \\
\text { anos e capacidade de } \\
\text { deambular sem auxílio. }\end{array}$ & Sim & $\begin{array}{c}\text { A precisão da previsão da } \\
\text { MOF (definida como uma } \\
\text { fratura no colo femoral, } \\
\text { coluna, antebraço distal ou } \\
\text { úmero proximal que ocorreu } \\
\text { após a linha de base) pode ser } \\
\text { melhorada incluindo o } \\
\text { software TBS, em } \\
\text { comparação com o FRAX® } \\
\text { sozinho em fraturas } \\
\text { osteoporóticas. }\end{array}$ \\
\hline $\begin{array}{c}\text { Lim et al., } \\
2016\end{array}$ & $\begin{array}{c}\text { Índice de comorbidades } \\
\text { utilizado para medição de } \\
\text { avaliação física e outro para } \\
\text { prever mortalidade. }\end{array}$ & Não & $\begin{array}{l}\text { As cirurgias de artroplastia } \\
\text { total de quadril devem ser } \\
\text { realizadas por cirurgiões de } \\
\text { quadris ou sob supervisão } \\
\text { direta para diminuição de }\end{array}$ \\
\hline
\end{tabular}




\begin{tabular}{|c|c|c|c|}
\hline & & & $\begin{array}{c}\text { complicações, morbidades e } \\
\text { mortalidades. }\end{array}$ \\
\hline $\begin{array}{c}\text { Maiche; } \\
\text { Hernández; } \\
\text { Mendoza, } \\
2019\end{array}$ & $\begin{array}{c}\text { Questionários com } \\
\text { comorbidades, localização e } \\
\text { topografia das fraturas, tempo } \\
\text { de resolução cirúrgica, } \\
\text { quedas, conhecimento do } \\
\text { diagnóstico de osteoporose, } \\
\text { tratamento para a doença, } \\
\text { fraturas anteriores e } \\
\text { posteriores, evolução do } \\
\text { paciente (institucionalização, } \\
\text { grau de deambulação e } \\
\text { mortalidade). }\end{array}$ & Sim & $\begin{array}{l}\text { Evidenciam a subestimação } \\
\text { do diagnóstico da } \\
\text { osteoporose, sendo fator } \\
\text { determinante das fraturas, os } \\
\text { indivíduos não recebem } \\
\text { tratamento adequado para } \\
\text { prevenir novos eventos. As } \\
\text { quedas são os precipitantes } \\
\text { das fraturas em quase todos os } \\
\text { casos, ocorrendo dentro de } \\
\text { suas próprias residências. }\end{array}$ \\
\hline $\begin{array}{l}\text { Rathbun et } \\
\text { al., } 2017\end{array}$ & $\begin{array}{c}\text { Dados de duas coortes; BHS- } \\
7 \text { para avaliar diferenças } \\
\text { sexuais nas sequelas de } \\
\text { fratura de quadril e a MOST } \\
\text { para diferenças raciais na } \\
\text { densidade mineral óssea de } \\
\text { homens mais velhos. Ambos } \\
\text { com varreduras do DXA. }\end{array}$ & Sim & $\begin{array}{c}\text { A Fratura de quadril em } \\
\text { idosos está associado a } \\
\text { declínios acelerados de } \\
\text { densidade mineral óssea } \\
\text { (DMO). Após a fratura do } \\
\text { quadril os declínios de DMO } \\
\text { em idosos foram maiores do } \\
\text { que a perda óssea ocorrida em } \\
\text { homens que não sofreram } \\
\text { fraturas. }\end{array}$ \\
\hline $\begin{array}{c}\text { Samelson et } \\
\text { al., } 2018\end{array}$ & $\begin{array}{l}\text { Questionários padronizados } \\
\text { para averiguação de fraturas, } \\
\text { incidentes por meio de } \\
\text { autorrelato dos participantes, } \\
\text { registros médicos ou } \\
\text { confirmação por profissionais } \\
\text { médicos, densitometria e } \\
\text { Frax. }\end{array}$ & Não & $\begin{array}{c}\text { As medidas periféricas de } \\
\text { densidade óssea cortical e } \\
\text { trabecular, microestrutura e } \\
\text { força estão associadas às } \\
\text { fraturas incidentes, porém } \\
\text { observaram que a maioria das } \\
\text { fraturas ocorreram em } \\
\text { indivíduos que não havia } \\
\text { osteoporose. }\end{array}$ \\
\hline
\end{tabular}




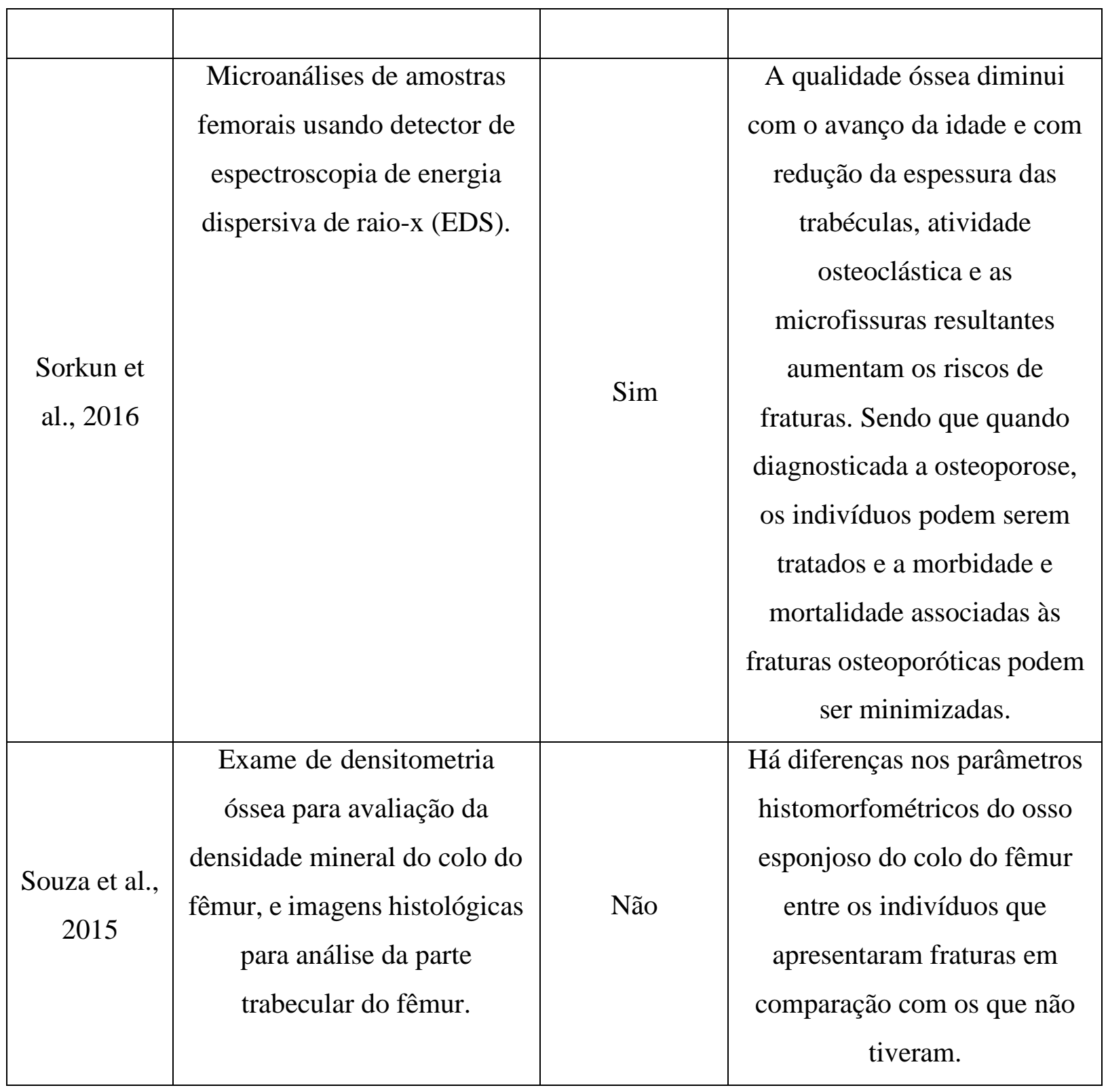

A densitometria por Absorciometria de Dupla Energia por Raios X (DEXA) é considerada o método padrão para avaliação da densidade mineral óssea. Consiste em um aparelho capaz de gerar duplo feixe de raio x que atravessa a região do corpo, pois a radiação emitida é obtida por um colimador que analisa a quantidade de cálcio pela área medida, o sistema é calibrado para expressar os resultados em gramas por centímetros quadrados (ANDRADE; SILVA; AMARAL, 2015).

Entretanto, a Fracture Risk Assessment Tool (FRAX) é uma ferramenta que associa o resultado da densidade mineral óssea com condições de riscos para fraturas em 10 anos por fragilidade óssea, a avaliação é realizada considerando o índice de massa corpórea, idade, sexo, históricos familiares de fraturas de fêmur, fraturas prévias, artrite reumatoide, osteoporose secundária e uso de corticoide prolongado (SILVA et al., 2016). 
No entanto, a Espectroscopia de Dispersão de Energia de Raios X (EDS) é baseada em um método de caracterização composicional que permite análise espectrográfica da composição das amostras para verificação de cálcio $(\mathrm{Ca})$ e fósforo $(\mathrm{P})$ nos locais de fraturas. Já o software TBS avalia o escore do osso trabecular e associa dados da densitometria óssea para análise, quanto aos parâmetros de volume ósseo, número de trabéculas e espaçamentos, aprofundando nas mudanças que ocorrem no arcabouço trabecular (GARCÍA et al., 2018; GLÜER, 2017; PALERMO et al., 2016; SORKUN et al., 2016).

Estudos de Maiche, Hernandez e Mendoza (2019) mostraram que apenas 15,22\% da população com diagnóstico de osteoporose sabia que eram portadores de tal patologia, porém alguns não tinham nem prescrições para tratamentos, e outros utilizavam de forma incompleta. Já $84,78 \%$ dos indivíduos tiveram conhecimento do diagnóstico após quedas e consequentes fraturas.

No esqueleto humano existem dois tipos de tecidos ósseos, o cortical e trabecular. O primeiro, apresenta tecido denso, compacto e com pouca porosidade, com a função de promover força mecânica e proteção. Já o segundo é encontrado na pelve e epífises de ossos longos, com alta porosidade. Sua remodelação óssea é superior ao cortical, devido ao maior número de células e por ser metabolicamente mais ativo, sendo crucial para detectar perca óssea, tornando o sítio anatômico alvo de monitorização e controle da osteoporose (WEAVER et al., 2016).

Estudos realizados na Turquia e Reino Unido demonstraram que ambos os gêneros podem ser afetados sem diferenças significativas. Entretanto, um no Uruguai, um no Norte da América e Europa, concluíram que o gênero feminino prevalece, principalmente com o avanço da idade. Porém, outro afirma que os homens experimentam declínios de densidade mineral óssea (DMO) com fraturas de quadril significativamente maiores (figura 1) (LIM et al., 2016; MAICHE; HERNÁNDEZ; MENDOZA, 2019; RATHBUN et al., 2017; SAMELSON et al., 2018; SORKUM et al., 2016). 
Figura 1- Gêneros estudados para detectar a prevalência da osteoporose, publicados entre os anos de 2015 e 2020.

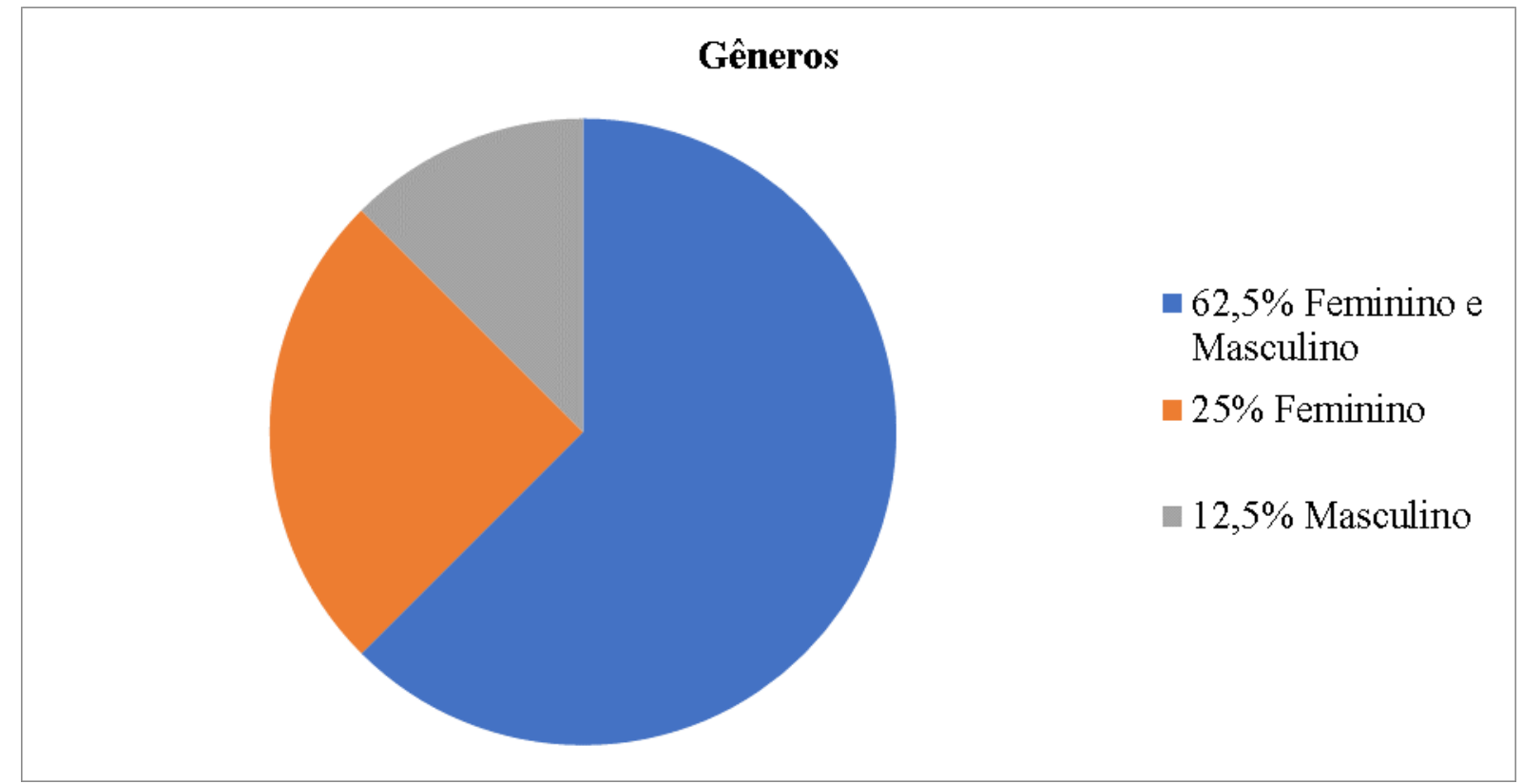

Fonte: Próprio autor.

Observa-se em ambos os gêneros a perca de massa óssea, sobretudo após os 65 anos, as mulheres são mais vulneráveis em decorrência da diminuição dos níveis de produção de estrogênio no ovário no período pós-menopausa, que causam destruição óssea, além do equilíbrio interrupto entre a composição óssea pelos osteoblastos e osteoclastos. O gênero masculino está menos suscetível a progredir com tal doença, devido ao nível superior de ganho de massa óssea ao longo da puberdade e menor agravo no envelhecer (BLACK e ROSEN, 2016; MELO et al., 2017; SILVA et al., 2015).

Um estudo realizado na Turquia constatou que a diminuição da densidade óssea, atividade osteoclástica e espessura das trabéculas aumentam as chances de fraturas, uma vez diagnosticada a osteoporose, essas pessoas podem ser tratadas e, desta forma, a morbidade e mortalidade associadas às fraturas osteoporóticas podem ser minimizadas (SORKUN et al., 2016).

A osteoporose é uma patologia relativamente silenciosa, assintomática até os primeiros episódios de fraturas, o que pode dificultar um diagnóstico precoce, o ideal seria iniciar desde a adolescência o consumo de cálcio, através da alimentação, realização de atividades físicas regularmente ou o uso de fármacos. Medidas simples que poderiam resultar no retardo da patologia e melhor qualidade de vida (DE ANDRADE, 2015; LIMA; MIRANDA; VASCONCELOS, 2017).

Nas análises realizadas no Canadá; $47 \%$ da população foi diagnosticada com osteoporose com base na densitometria óssea, sendo que 30\% sofreram um ou mais episódios 
de quedas, e $17 \%$ tiveram fraturas no quadril. O risco e fraturas de idosas com osteoporose, baixa massa óssea ou histórico de fratura é maior entre aquelas com histórico de quedas, de fratura por baixo trauma e pior função física (ADACHI et al., 2019).

Os incidentes de quedas ocorridas da própria altura nas observações de Samelson e colaboradores (2018) estavam presentes em 67\% dos casos, sendo responsáveis por $9 \%$ das fraturas de quadril. E os fatores que foram associados, estão a idade senil, gênero feminino, uso de medicação para osteoporose, fraturas anteriores, maior pontuação Frax e menor pontuação T na densitometria óssea.

Em 94,57\% dos casos de fraturas, $81,52 \%$ equivalem as do colo femoral, e a cirurgia de artroplastia com implante protético foi o método escolhido, já 18,48\% na região trocantérica com realização de osteossíntese, havendo um atraso no processo cirúrgico com uma mediana de tempo de 9 e 11 dias respectivamente. Os principais objetivos das cirurgias são de auxiliar na restauração da anatomia da região e proporcionar recuperação funcional do membro (MAICHE; HERNÁNDEZ; MENDOZA, 2019; RAMALLO et al., 2019).

Entretanto, um estudo no Uruguai averiguou que $66,43 \%$ dos pacientes necessitaram de cuidados domiciliares com suporte instrumental e 77,72\% retornaram à deambulação, pois nem sempre é atingido uma recuperação completa o que tornam os indivíduos dependentes para a realização das atividades de vida diária (AVD’s). As taxas de mortalidade chegaram a $11 \%$ dentro de um ano após fratura (CANÇADO et al., 2015; MAICHE; HERNÁNDEZ; MENDOZA, 2019).

As mortalidades de pacientes com fraturas do colo femoral ocorrem em razão ao tempo de internação e a demora para realização das cirurgias, com maiores chances de infecções hospitalares. O tempo de internação pode ser indicador para desordens pósoperatórias como infecções nos locais da cirurgia, trombose venosa profunda, embolia pulmonar e pneumonia, podendo induzir a morte (CORREA et al., 2020; LEHTONEN et al., 2018; LIZAUR-UTRILLA et al., 2019).

Há uma diminuição no tempo de internação e mortalidade quando as cirurgias por fraturas de fêmur em idosos são realizadas em até 48 horas após a internação hospitalar. Porém, o tratamento cirúrgico ainda tem controvérsias, devido aos aspectos que interferem na decisão como, comorbidades clínicas, disponibilidade das equipes e de materiais (PINTO et al., 2019).

\section{CONCLUSÃO}


No intuito de verificar a osteoporose como uma preditora de fraturas do colo femoral em decorrência de quedas na população idosa, verificou-se que a presença desta patologia é considerada um fator predisponente para fraturas por quedas, porém observa-se uma limitação no diagnóstico e tratamento precoce por ser uma doença assintomática e silenciosa. Tais fraturas foram provenientes de quedas e o gênero feminino foi predominante, devido à uma diminuição nos níveis de produção de estrogênio no ovário no período pós-menopausa causando destruição óssea.

Os procedimentos cirúrgicos constituem o tratamento mais utilizado, porém a demora no tempo de internação e a espera pela cirurgia podem acarretar comprometimentos funcionais aos indivíduos e aumentar a mortalidade. A produção científica entre o período estabelecido de 2015 e 2020 especificamente em idosos e no colo femoral é escassa. Recomendam-se mais estudos com essas populações para maiores investigações.

\section{REFERÊNCIAS BIBLIOGRÁFICAS}

ADACHI, J. D. et al. Predictors of imminent non-vertebral fracture in elderly women with osteoporosis, low bone mass, or a history of fracture, based on data from the population-based Canadian Multicentre Osteoporosis Study (CaMos). Archives of osteoporosis, v. 14, n. 1, p. 53, 2019. Disponível em: 〈https://link.springer.com/article/10.1007/s11657-019-0598-X > Acesso em: 30 set. 2020.

ANDRADE, S.R.S.; SILVA, M.R.S.; AMARAL, W.N. Propedêutica diagnóstica da osteoporose. Femina, v.43, n. 3, p. 135-148, 2015. Disponível em:

<http://files.bvs.br/upload/S/01007254/2015/v43n3/a5122.pdf > Acesso em: 17 set. 2020.

BARRETO, M.S.; CARREIRA, L.; MARCON, S.S. Envelhecimento populacional e doenças crônicas: Reflexões sobre os desafios para o Sistema de Saúde Pública. Revista Kairós: Gerontologia, v. 18, n. 1, p. 325-339, 2015. Disponível em:

<http://ken.pucsp.br/kairos/article/view/26092> Acesso em: 07 ago. 2020.

BLACK, D.M.; ROSEN, C.J. Postmenopausal osteoporosis. New England Journal of Medicine, v. 374, n. 3, p. 254-262, 2016. Disponível em:

<https://www.osteostrategy.on.ca/wp-content/uploads/Black-D.-Rosen-C.J.-20161.pdf >

Acesso em: 12 out. 2020.

CANÇADO, B.L. et al. Importância da avaliação óssea e da prevenção da fratura osteoporótica em pacientes com câncer de proìstata em uso de anaìlogos do hormônio gonadotrófico. Revista do Colégio Brasileiro de Cirurgiões, v. 42, n. 1, p. 62-66, 2015. Disponível em: 〈https://www.scielo.br/pdf/rcbc/v42n1/pt_0100-6991-rcbc-42-01 00062.pdf> Acesso em: 09 set. 2020. 
CESAR, R. et al. Relating mechanical properties of vertebral trabecular bones to osteoporosis. Computer Methods in Biomechanics and Biomedical Engineering, v. 23, n. 2, p. 54-68, 2020. Disponível em: 〈https://scihub.tw/https://doi.org/10.1080/10255842.2019.1699542> Acesso em: 07 ago. 2020.

CORREA, J.G.L. et al. Evaluation of predictive factors of in hospital mortality in patients with proximal femoral fracture. Acta Ortopédica Brasileira, v. 28, n. 1, p. 40-43, 2020. Disponível em:

<https://www.scielo.br/scielo.php?pid=S141378522020000100040\&script=sciarttext $>$ Acesso em: 04 out. 2020.

CURTIS, E.M. et al. Reprint of: the impact of fragility fracture and approaches to osteoporosis risk assessment worldwide. Inter. Journal of Orthopaedic and Trauma Nursing, v. 26, p. 7-17, 2017. Disponível em:

<https://www.sciencedirect.com/science/article/abs/pii/S1878124117300370> Acesso em: 17 ago. 2020.

DE ANDRADE, S.A.F. Osteoporose: um problema de saúde pública. UNILUS Ensino e Pesquisa, v. 12, n. 28, p. 41-47, 2015. Disponível em:

<http://revista.lusiada.br/index.php/ruep/article/view/399> Acesso em: 14 set. 2020.

EDELMUTH, S.V.C.L. et al. Comorbidades, intercorrências clínicas e fatores associados à mortalidade em pacientes idosos internados por fratura de quadril. Revista Brasileira de Ortopedia, v. 53, n. 5, p. 543-551, 2018. Disponível em:

<https://www.sciencedirect.com/science/article/pii/S0102361617303958> Acesso em: 04 ago. 2020 .

GALVÃO, T. F.; PANSANI, T. S. A.; HARRAD, D. Principais itens para relatar Revisões sistemáticas e Meta-análises: A recomendação PRISMA. Epidemiologia e Serviços de Saúde, v. 24, p. 335-342, 2015. Disponível em:

<https://www.scielosp.org/article/ress/2015.v24n2/335-342/pt/> Acesso em: 29 set. 2020.

GARCÍA, F. N. J. et al. Obtención y caracterización de recubrimientos de Brushita, Dióxido de Titanio (TiO2) y sus bicapas sobre acero 316L. Entre Ciência e Ingeniería, v. 12, n. 23, p. 131-139, 2018. Disponível em:

<https://revistas.ucp.edu.co/index.php/entrecienciaeingenieria/article/view/25> Acesso em: 28 nov. 2020.

GLÜER, C.C. 30 years of DXA technology innovations. Bone, v. 104, p. 7-12, 2017. Disponível em: <https://www.sciencedirect.com/science/article/abs/pii/S8756328217301862> Acesso em: 28 nov. 2020. 
HAGINO, H. et al. Survey of hip fractures in Japan: recent trends in prevalence and treatment. Journal Orthopedic Science, v.22, p. 909-914, 2017. Disponível em:

$<$ https://www.sciencedirect.com/science/article/pii/S0949265817301641> Acesso em: 01 set. 2020 .

IKI, M. et al. Trabecular bone score may improve FRAX® prediction accuracy for major osteoporotic fractures in elderly Japanese men: the Fujiwara-kyo Osteoporosis Risk in Men (FORMEN) Cohort Study. Osteoporosis International, v. 26, n. 6, p. 1841-1848, 2015. Disponível em: <https://link.springer.com/article/10.1007/s00198-015-3092-3> Acesso em: 28 out. 2020.

LEHTONEN, E.J.I. et al. Trends in surgical treatment of femoral neck fractures in the elderly. Einstein (São Paulo), v. 16, n. 3, p. 1-7, 2018. Disponível em: $<$ https://www.scielo.br/pdf/eins/v16n3/pt_1679-4508-eins-16-03-eAO4351.pdf $>$ Acesso em: 10 set. 2020.

LIM, Jun Wei et al. Total hip replacement for neck of femur fracture: Comparing outcomes with matched elective cohort. Injury, v. 47, n. 10, p. 2144-2148, 2016. Disponível em: <https://www.sciencedirect.com/science/article/abs/pii/S0020138316302972> Acesso em: 27 out. 2020.

LIMA, A.L.C.; MIRANDA, S.C.; VASCONCELOS, H.F.O. Anatomia radiográfica do fêmur proximal: fratura de colo vs. fratura transtrocantérica. Revista Brasileira de Ortopedia, v. 52, n. 6, p. 651-657, 2017. Disponível em: <https://www.scielo.br/pdf/rbort/v52n6/pt_19824378-rbort-5206-00651.pdf > Acesso em: 18 set. 2020.

LIU, W. et al. Meta-analysis of osteoporosis: fracture risks, medication and treatment. Minerva Medicina, v.106, n. 4, p. 203-214, 2015. Disponível em: <https://pubmed.ncbi.nlm.nih.gov/26125152/> Acesso em: 03 set. 2020.

LIZAUR-UTRILLA, A. et al. Reasons for delaying surgery following hip fractures and its impact on one year mortality. International Orthopaedics, v. 43, n. 2, p. 44-448, 2019. Disponível em: 〈https://www.ncbi.nlm.nih.gov/pubmed/29744645> Acesso em: 01 out. 2020.

MAICHE, M.; HERNÁNDEZ, M.; MENDOZA, B. Características y evolución de las fracturas de cadera operadas en el Banco de Prótesis (enero-diciembre 2013). Revista Médica del Uruguay, v. 35, n. 3, p. 82-106, 2019. Disponível em:

$<$ http://www.scielo.edu.uy/pdf/rmu/v35n3/1688-0390-rmu-35-03-82.pdf $>$ Acesso em: 26 set. 2020. 
MELO, A.C.F. et al. Prevalência de doenças musculoesqueléticas autorreferidas segundo variáveis demográficas e de saúde: estudo transversal de idosos de Goiânia/GO. Cadernos Saúde Coletiva, v. 25, n. 2, p. 138-143, 2017. Disponível em:

<https://www.scielo.br/pdf/cadsc/v25n2/1414-462X-cadsc-25-2-138.pdf> Acesso em: 12 set. 2020 .

MUNHOZ, L. et al. Bone mineral density and mandibular osteoporotic alterations in type 2 diabetes. Brazilian Dental Science, v. 21, n. 2, p. 220-229, 2018. Disponível em:

<https://ojs.ict.unesp.br/index.php/cob/article/view/1587/1246> Acesso em: 15 out. 2020.

NETO, S.M et al. Proximal disability and spinal deformity index in patients with proximal femur fractures. Coluna/Columna, v. 14, n. 4, p. 276-280, 2015. Disponível em: $<$ https://www.scielo.br/scielo.php?pid=S180818512015000400276\&script=sciarttext $>$ Acesso em: 18 ago. 2020.

PALERMO, A. et al. BMI and BMD: the potential interplay between obesity and bone fragility. International journal of environmental research and public health, v. 13, n. 6, p. 544, 2016. Disponível em: 〈https://www.mdpi.com/1660-4601/13/6/544> Acesso em: 28 nov. 2020.

PINTO, I.P. et al. Does Early Surgical Fixation of Proximal Femoral Fractures in Elderly Patients Affect Mortality Rates? Revista Brasileira de Ortopedia, v. 54, n. 4, p. 392-395, 2019. Disponível em: <https://www.scielo.br/pdf/rbort/v54n4/pt_1982-4378-rbort-54 04392.pdf> Acesso em: 31 ago. 2020.

RAMALLO, D.A. et al. Fatores que influenciam o resultado da osteossíntese na fratura do colo do fêmur em pacientes adultos jovens. Revista Brasileira de Ortopedia, v. 54, n. 04, p. 408-415, 2019. Disponível em:

〈https://www.thiemeconnect.com/products/ejournals/pdf/10.1016/j.rbo.2017.12.021.pdf $>$

Acesso em: 29 set. 2020.

RATHBUN, A. M. et al. Older men who sustain a hip fracture experience greater declines in bone mineral density at the contralateral hip than non-fractured comparators. Osteoporosis International, v. 29, n. 2, p. 365-373, 2017. Disponível em: <https://link.springer.com/article/10.1007/s00198-017-4280-0> Acesso em: 04 out. 2020.

SAMELSON, E. J. et al. Cortical and trabecular bone microarchitecture as an independent predictor of incident fracture risk in older women and men in the Bone Microarchitecture International Consortium (BoMIC): a prospective study. The lancet Diabetes $\boldsymbol{\&}$ endocrinology, v. 7, n. 1, p. 34-43, 2018. Disponível em: <https://www.sciencedirect.com/science/article/abs/pii/S2213858718303085> Acesso em: 26 out. 2020 . 
SCHURMAN, L. et al. Guías Argentinas para el Diagnóstico, la Prevención y el Tratamiento de la Osteoporosis 2015. Actual Osteoporosis, v. 13, n. 2, p.136-156, 2017. Disponível em: <https://digital.cic.gba.gob.ar/handle/11746/6120> Acesso em: 01 set. 2020.

SILVA, A.C.V. et al. Fatores associados à osteopenia e osteoporose em mulheres submetidas à densitometria óssea. Revista Brasileira de Reumatologia, v. 55, n. 3, p. 223-228, 2015. Disponível em: 〈https://www.sciencedirect.com/science/article/pii/S0482500414002095> Acesso em: 12 mar. 2020.

SILVA, C.S.O. et al. Family health strategy: relevance to the functional capacity of older people. Revista Brasileira de Enfermagem, v. 71, p. 740-746, 2018. Disponível em: $<$ https://www.scielo.br/scielo.php?pid=S003471672018000800740\&script=sci_artte\&tlng=pt $>$ Acesso em: 06 ago. 2020.

SILVA, M.R.S.; ANDRADE, S.R.S.; AMARAL, W.N. Fisiopatologia da osteoporose: uma revisão bibliográfica. Femina, v. 43, n.6, p. 241-244, 2015. Disponível em: < a5322.pdf (bvs.br) > Acesso em: 06 set. 2020.

SILVA, Y.B. et al. Correlation between osteoporotic fracture risk in Brazilian postmenopausal women calculated using the FRAX with and without the inclusion of bone densitometry data. Archives Osteoporosis, v.11, n.1, p.1-7, 2016. Disponível em: <https://scihub.wikicn.top/https://doi.org/10.1007/s11657-015-0255-y > Acesso em: 22 set. 2020 .

SINGER, A. et al. Burden of illness for osteoporotic fractures compared with other serious diseases among postmenopausal women in the United States. Mayo Clinic Proceedings, v. 90, n. 1, p.53-62, 2015. Disponível em:

<https://cdn.nof.org/wpcontent/uploads/2016/01/1047.pdf> Acesso em: 08 set. 2020.

SORKUN, H. Ç. et al. Assessment of femoral neck fractures in the elderly with respect to morphology and mineral density. Folia morphologica, v. 75, n. 4, p. 536-542, 2016. Disponível em: 〈https://journals.viamedica.pl/folia_morphologica/article/view/44086> Acesso em: 01 nov. 2020.

SOUZA, C.G. et al. Histomorphometric bone assessment in patients with fracture of the proximal end of the femur. Acta Ortopedica Brasileira, v. 23, n. 2, p. 103-106, 2015. Disponível em: <https://www.scielo.br/scielo.php?pid=S141378522015000200103\&script=sciarttext $>$ Acesso em: 18 ago. 2020. 
SPINELLI, L.F. et al. Comparação clínica, laboratorial e densitométrica de pacientes com coxartrose e com fraturas do colo femoral. Revista do Colégio Brasileiro de Cirurgiões, v. 45, n. 5-33, 2018. Disponível em:

<https://www.scielo.br/scielo.php?pid=S010069912018000500156\&script=sciarttext $>$

Acesso em: 20 ago. 2020.

TOMMASO, A. B. G. D. et al. Geriatria - Guia Prático. 1.ed. São Paulo: Guanabara Koogan, 2016.

VALLES-FIGUEROA, J.F. et al. Estudio comparativo de los eventos adversos entre el abordaje posterolateral y lateral directo para artroplastía primaria de cadera no cementada en pacientes mayores de 65 años con fracturas del cuello femoral. Acta Ortopédica Mexicana, v. 29, n. 1, p. 1-12, 2015. Disponível em:

<http://www.scielo.org.mx/scielo.php?pid=S230641022015000100001\&script=sciarttext $>$

Acesso em: 26 ago. 2020.

WEAVER, C.M. et al. The National Osteoporosis Foundation's position statement on peak bone mass development and lifestyle factors: a systematic review and implementation recommendations. Osteoporosis international, v. 27, n. 4, p. 1281-1386, 2016. Disponível em: <https://link.springer.com/article/10.1007/s00198-015-3440-3> Acesso em 05 ago. 2020. 